\title{
Relationship between innovative and biosimilar trastuzumab used in the treatment of breast cancer: cross-sectional study of financial impact
}

\begin{abstract}
The irrational use of drugs today is relevant, so pharmacoeconomics is an analytical tool that links economic factors related to drug use and clinical outcomes. Biological medicines are products derived from genetically modified living cell sources. In this context, the biosimilar drug presents similarity in structure, function, efficacy and safety when compared to the original product. Currently several biosimilars are being developed for the treatment of breast cancer, a fact that directly impacts patient survival. Breast cancer is one of the most common types of cancer, being HER2 positive one of the most aggressive forms of the disease, and its treatment is based on the use of cytotoxic drugs associated with targeted therapy using the monoclonal antibody transtuzumab. There are positive future prospects related to biosimilars with impacts on survival and potential increase of patients with access to innovative treatments. The research aimed to analyze the financial impact of the use of the biosimilar trastuzumab and the increase in the number of patients with access to therapies, through a retrospective documentary cross-sectional study, developed on a representative sample of cost values of the drugs Herceptin and Zedora, for the reference protocol trastuzumab. The results obtained in the descriptive statistics through the analysis of variance were not significant; however, a percentage of savings of $27.89 \%$ was obtained for the biosimilar Zedora when compared to Herceptin. Monoclonal antibodies are responsible for $35 \%$ of the expenses in oncology. The introduction of biosimilars can contribute to the financial and budgetary sustainability of the health system. Based on the results obtained, it is possible to affirm that for every three patients treated with Herceptin, it is possible to treat four patients using Zedora.
\end{abstract}

Keywords: pharmacoeconomics, biological medications, biosimilar medications, indication extrapolation, interchangeability
Volume 12 Issue 4 - 202I

\author{
Tamara Marques Previ,Andre Fellipe Freitas \\ Rodrigues \\ Universidade do Oeste Paulista, Brazil
}

Correspondence: Tamara Marques Previ, Universidade do Oeste Paulista - Unoeste, Presidente Prudente - SP, Brazil, Email tamaraprevi@hotmail.com

Received: July 13, 2021 | Published: July 29, 2021

\section{Introduction}

The irrational use of drugs plays an important role in the context of current public health problems, thus, the development of measures aimed at pharmacotherapeutic safety and effectiveness were necessary. In this context, pharmacoeconomics is an analytical tool that associates economic factors related to the use of drugs and their clinical outcomes, represents an important tool in the management of pharmaceutical products in order to improve the use of drugs, reduce the risks of morbidity and mortality and costs related to pharmacotherapy.

Biological drugs are widely used in the treatment of chronic diseases, including diabetes, rheumatoid arthritis, multiple sclerosis and cancer. They are products derived from genetically modified live cell sources through recombinant DNA technology or by hybridoma production, providing the production of proteins for therapeutic purposes. Therefore, due to their elaborate means of production, these drugs have much more complex characteristics than drugs obtained by chemical synthesis. ${ }^{2}$

The differences between biological and synthetic medicines are not limited in origin, they differ in several aspects. Generally, synthetic drugs have small molecules, made up of tens or a few hundred atoms and a simple and well-known chemical structure; due to their nature they are stable molecules, consequently enabling identical replicas of their chemical formula. Unlike synthetics, biological medicines have large, complex molecules made up of thousands of atoms; they are usually unstable and susceptible when subjected to small variations in conservation and storage conditions, thus making identical copies impossible. Its administration routes are injectable and inhalable, as they are inactivated by digestive enzymes, so oral options are rare. ${ }^{3}$

According to the Ministry of Health, ${ }^{4}$ in its Law No. 9,797, it defined a generic drug as an identical copy of a reference drug already registered with the competent body after patent expiration, produced by chemical synthesis, with proven interchangeability through a bioavailability test and bioequivalence. Thus, through these tests, it is possible to prove the therapeutic equivalence of generic drugs, as well as their safety and clinical efficacy.

Similar to the generic drug, the development of biossiomilar is only possible after the patent of the reference product expires. However, the biosimilar medicine is not a generic medicine, but a version of a reference biological medicine with similar molecular structure, function, efficacy and safety. The use of these agents in the treatment of diseases such as cancer requires a cautious approach to ensure there are no negative impacts from their use on the patient. ${ }^{5}$

The interchangeability of a biological reference product for a biossiomilar is only possible if there is no loss of efficacy or safety reduction. ${ }^{6}$ According to ANVISA, ${ }^{7}$ in line with current WHO recommendations and recognized international guides from other regulatory agencies, specific studies for the demonstration of interchangeability are not mandatory for the approval of a biosimilar. Biological drugs often have more than one therapeutic indication, 
however the extrapolation of indication of the reference biological drug is only possible if the biosimilar drug presents the same mechanism of action and receptor involved for such indications, proven through tests of comparability and adequate justification. ${ }^{8}$ Currently, several biosimilars are being developed for the treatment of breast cancer, a fact that directly impacts the patient's survival and raises several questions. ${ }^{9}$

Breast cancer is one of the most common types of cancer, although advances in diagnosis and treatment have significantly reduced mortality rates, it still stands out as the second leading cause of death in the female population. Among the various forms of the disease, the expression of human epidermal growth factor receptor 2 (HER2) is particularly one of the most aggressive forms of breast cancer, called HER2 positive. The HER2 receptor is a transmembrane protein tyrosine kinase responsible for controlling the division and repair of breast cells during the cell cycle. The treatment of this form of the disease is based on the use of standard chemotherapy with cytotoxic drugs associated with the target therapy using the monoclonal antibody transtuzumab. ${ }^{10}$ There are several concerns about the expansion of biosimilars and the safety of their use, immunogenicity is identified by the European Agency regulations as the main one. ${ }^{11}$

Immunogenicity is the ability to activate an immune reaction or response by insoluble forms of the drug that can trigger an autoreactive immune response, or even the presence of contaminants, denaturation of proteins and endotoxins in the preparation of the drug, which can cause the development of specific antibodies, sensitization of $\mathrm{T}$ cells and allergic/anaphylactic reactions. Variations in the type of protein that the drug presents guides the potential for developing an immune reaction, the greater the similarity between the biological product and the human protein, the lower the probability of developing immunogenic reactions; An example of this variation of similarity to the human protein are the monoclonal antibodies, which are subdivided into four types: murine, chimeric, humanized and human. ${ }^{12}$

Pharmacovigilance is the science and activities related to the verification, understanding, evaluation and prevention of adverse effects or which problems concerning the use of medications. Thus, due to the complexity of producing biosimilars, and considering that minor molecular changes can cause serious reactions, the implementation of risk management and pharmacovigilance programs is of paramount importance. ${ }^{13}$

\section{Justification}

The advancement and incorporation of new technologies in the health area have led to cost increases, so the financial sustainability of health resources is currently a matter of concern. ${ }^{14}$ The incorporation of innovative drugs, including biological drugs, especially monoclonal antibodies, are among the main factors related to rising costs. ${ }^{15}$ There are positive future perspectives regarding the expansion of the development of biosimilars, leading to a phase of interests focused on cost reduction and pharmacoeconomics, allowing the potential increase in the number of patients with access to innovative treatments. ${ }^{14}$

\section{Objective}

Analyze the financial impact of the use of biosimilar Transtuzumab in the current cancer context and the increase in the number of patients with access to therapies.

\section{Methodology}

This is a cross-sectional retrospective documental study, developed in a representative sample of cost values of the medicines Herceptin (innovative biological medicine) and Zedora (biosimilar medicine). The cost values considered were selected by systematic sampling, from October 2017 to September 2018, based on a list obtained through the proprietary electronic information system and ordered by months and medication used, in a private treatment unit of chemotherapy from the interior of SP. Cost values were considered for the transtuzumab reference protocol, loading dose $8 \mathrm{mg} / \mathrm{kg}$ and maintenance dose $6 \mathrm{mg} /$ $\mathrm{kg}$, repetition every 22 days, continuous administration for 12 months of treatment totaling 17 cycles, applied to patients between 72 and 91 kilograms. ${ }^{16}$

\section{Results}

Thus, based on the information obtained, there are the following graphs referring to costs for the Transtuzumab reference protocol (Figure 1):

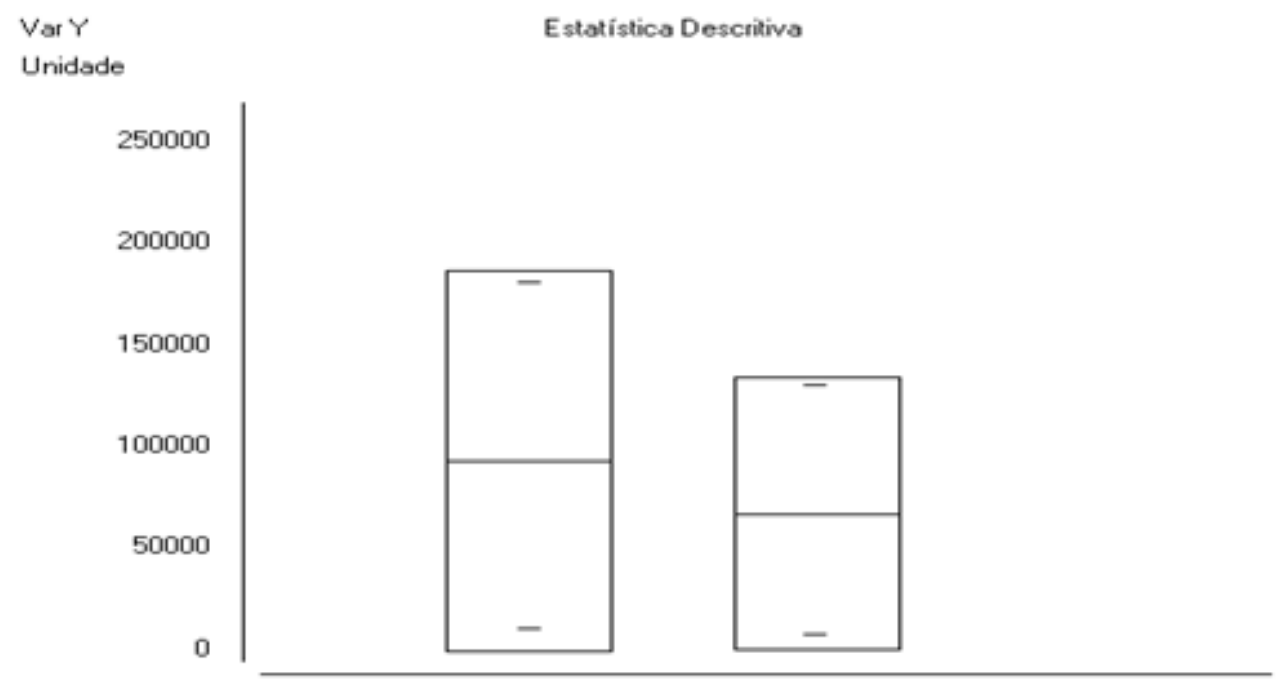

Figure I Descriptive cost statistics for Herceptin and Zedora drugs. 
The chart above shows the descriptive cost statistics of the innovative biological medicine Herceptin and its biosimilar Zedora. In the analysis of variance, there was no significance: sources of variation $\mathrm{gl} \mathrm{sq}$ qm; treatments $113.4 \mathrm{e}+0813.4 \mathrm{e}+08$; error 639.8 e+09 66.4 e+08; F=0.2023; $(p)=0.6701$ (Figure 2).

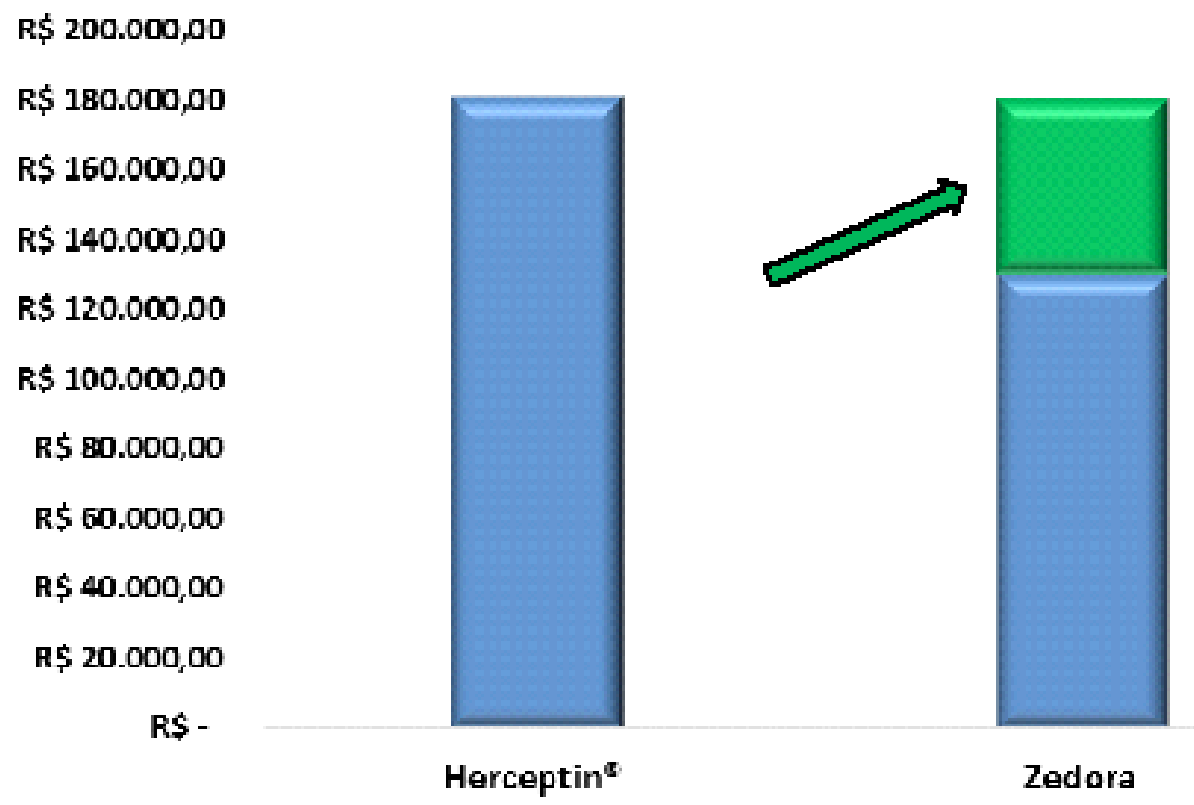

Figure 2 Total cost of the Transtuzumab reference protocol according to the medication used.

The graph above shows the total cost for the Transtuzumab reference protocol according to the medication used (innovative biological/biosimilar), loading dose $8 \mathrm{mg} / \mathrm{kg}$ and maintenance dose $6 \mathrm{mg} / \mathrm{kg}$, repeated every 22 days, for a total of 17 cycles. $^{16}$ The average weight found in the study of $78 \mathrm{~kg}$ was used. The cost values for the month of September 2018 were considered. Through the difference in the total cost value for the medications used in the study, the percentage of savings of $27.89 \%$ was obtained for the biosimilar medicine Zedora.

\section{Discussion}

Cancer treatment has a significant weight on the health system budget, the increase in life expectancy and population aging directly influence the continuous increase in the number of cancer cases. Improvements in the diagnosis and treatment of the disease are proportional to the increase in the permanence and commitment of patients to the treatments. In 2014, global spending on Oncology reached $\$ 100$ billion, with targeted therapy spending accounting for nearly $50 \%$ of that total. Monoclonal antibodies account for $35 \%$ of oncology spending, in 2015 the US spent 5.6 billion dollars. ${ }^{17}$

According to the results obtained in descriptive statistics, analyzing the variance, a standard deviation of 0.6701 is perceived, so there was no significant cost variation between the analyzed medications Herceptin and Zedora. For Renwick et al. ${ }^{17}$, the discrepancy in the values of biosimilars is associated with the high development costs and the absence of direct competition between this product and its reference product, currently the cost value of the biosimilar medicine ranges from around $70 \%$ to $85 \%$ in relation to the cost value of the innovative reference medicine.

According to Scheinberg et al., ${ }^{18}$ the development process of biological medicines is costly and complex, as it is based on the production of large and complex molecules by living organisms, whose small changes in the generation and execution of the process can directly affect the efficacy and safety. Grabowski et al., ${ }^{19}$ estimate that the costs involved with research and development of biosimilars vary between 10 and 40 million dollars, and the investments for their production vary between 250 and 450 million dollars.

On the other hand, Jackson ${ }^{15}$ states that even though monoclonal antibodies have a high cost, the approval of new drugs has increased year after year, representing one of the main factors responsible for the rise in health expenditure. For Gomes $\&$ Jardim, ${ }^{12}$ the clinical use of monoclonal antibodies in their various indications results in an increase in the survival rate and an improvement in the quality of life of patients.

Cortés et al., ${ }^{5}$ highlight that the introduction of Transtuzumab in patients with metastatic disease, previously untreated with the monoclonal antibody, resulted in an increase in median overall survival of 4.8 months; with regard to early-stage breast cancer, treatment with Transtuzumab for one year resulted in a significant reduction in the risk of disease recurrence.

Regarding the results obtained in the total cost analysis for the reference protocol Transtuzumab using different medications, the innovative biological medicine Herceptin and its biosimilar Zedora, it is clear that both medications have a high total cost, however, an important economy is highlighted. $27.89 \%$ related to the use of Zedora compared to the use of Herceptin. According to Zelenetz, ${ }^{20}$ the current estimate is that the biosimilar medicine costs $20 \%$ to $40 \%$ cheaper than the reference medicine.

A pioneer in the biosimilars market, on the European continent the use of these medicines provided savings of 20 to $30 \%$ compared to reference biological medicines. ${ }^{12}$ The introduction of biosimilars can significantly contribute to the financial and budgetary sustainability 
of the health system, there is a projected saving of 25 million dollars in drug costs by $2018 .{ }^{21}$ According to Jacobs et al., ${ }^{22}$ the expansion of the use of biosimilars, in addition to considerably alleviating the costly impact of these medications on health systems, could increase patients' access to innovative treatments.

The biosimilars market represents for Brazil, as well as for other emerging countries, a promising perspective from the economic point of view for the laboratories that manufacture this medication, patients and payers, including the Federal Government.

\section{Final considerations}

It is indisputable that innovative cancer treatments cultivate positive perspectives regarding the prognosis of the disease. The advent of biological medicines raises interest in the expansion and dissemination of treatments involving monoclonal antibodies, however expenditure on oncology is significantly high, with targeted therapies accounting for almost half of this amount. The expiration of patents on innovative biological medicines allowed the emergence of biosimilars, which, despite having a high cost and representing a financial impact on the health system, allows for important savings in relation to the reference medicine. Based on the results obtained in the study, it is possible to state that for every three patients treated with the drug Herceptin, it is possible to treat four patients using its biosimilar Zedora. Therefore, it is concluded that the development of a structured pharmacoeconomics makes it possible to increase the number of patients with access to innovative therapies, consequently increasing the survival rate and providing an improvement in the quality of life of patients diagnosed with HER2 positive breast cancer

\section{Acknowledgments}

None.

\section{Conflicts of interest}

Authors declare that there is no conflict of interest.

\section{References}

1. Aguiar KS, Santos JM, Cambrussi MC, et al. Patient safety and the value of pharmaceutical intervention in a cancer hospital. Einstein (Sao Paulo). 2018;16(1):eAO4122.

2. Declerck PJ. Biologicals and biosimilars: a review of the science and its implications. Generics and Biosimilars Initiative Journal. 2012;1(1):1316

3. Interfarma. Entendendo os Medicamentos Biológicos. São Paulo: Associação Brasileira da Indústria Farmacêutica de Pesquisa; 2012. 28 p.

4. Ministério da saúde. Lei $n^{\circ} 9.787$, de 10 de fevereiro de 1999. Altera a Lei no 6.360, de 23 de setembro de 1976, que dispõe sobre a vigilância sanitária, estabelece o medicamento genérico, dispõe sobre a utilização de nomes genéricos em produtos farmacêuticos e dá outras providências. Diário Oficial da União: 1, Brasília, DF; 1999.
5. Cortés J, Curigliano G, Diéras V. Expert perspectives on biosimilar monoclonal antibodies in breast cancer. Breast Cancer Research and Treatment. 2014;144(2):233-239.

6. Mellstedt H. Anti-neoplastic biosimilars-the same rules as for cytotoxic generics cannot be applied. Annals of Oncology. 2013;24(Suppl 5):23-28.

7. ANVISA. Nota de Esclarecimento $n^{\circ} 003 / 2017 / G P B I O / G G M E D /$ ANVISA-REVISADA. Gerência de Avaliação de Produtos Biológicos. Brasília, DF: Agência Nacional De Vigilância Sanitária; 2018.

8. Curigliano G, O'Connor DP, Jacobs I, et al. Biosimilars: Extrapolation for oncology. Critical Reviews in Oncology/Hematology. 2016;104:131-137.

9. European Medicines Agency. Guideline on similar biological medicinal products containing monoclonal antibodies-non-clinical and clinical issues. CHMP/BMWP/403543/2010. London: EMA; 2010.

10. Wilson FR, Coombes ME, Wylie Q, et al. Herceptin ${ }^{\circledR}$ (trastuzumab) in HER2-positive early breast cancer: Protocol for a systematic review and cumulative network meta-analysis. Systematic Reviews. 2017;6(1):196.

11. Schellekens H, Smolen JS, Dicato M, et al. Safety and efficacy of biosimilars in oncology. Lancet Oncology. 2016;17(11):e502-e509.

12. Gomes GAT, Jardim DLF. Biossimilares. 1st ed. Rio de Janeiro: Elsevier; 2017.

13. Camacho LH, Frost CP, Abella E, et al. Biosimilars 101: Considerations for U.S. oncologists in clinical practice. Cancer Medicine. 2014;3(4):889899.

14. Henry D, Taylor C. Pharmacoeconomics of Cancer Therapies: Considerations w the Introduction of Biosimilars. Seminars in Oncology. 2014;41(Suppl 3):S13-S20.

15. Jackson DB. Personalized cancer medicine - advances and socioeconomic challenges. Nat Rev Clin Oncol. 2012;8(12):735-741.

16. Kuehr T, Thaler J, Woell E. Chemotherapy Protocols 2018: Current Protocols and "Targeted Therapies". 19th ed. Wels and Zams: Innsbruck; 2018

17. Renwick MJ, Smolina K, Gladstone EJ, et al. Postmarket policy considerations for biosimilar oncology drugs. Lancet Oncology. 2016;17(1):e31-e38.

18. Scheinberg MA, Felix PA, Kos IA, et al. Partnership for productive development of biosimilar products: perspectives of access to biological products in the Brazilian market. Einstein (Sao Paulo). 2018;16(3):eRW4175

19. Grabowski HG, Ridley DB, Schulman KA. Entry and Competition in Generic Biologics. Manage Decis Econ. 2007;28(4-5):439-451.

20. Zelenetz AD. Biosimilars in Oncology. Oncology \& Hematology Review. 2016;12(1):22-28.

21. Ventola CL. Evaluation of Biosimilars for Formulary Inclusion: Factors for Consideration by P\&T Committees. P T. 2015;40(10):680-689.

22. Jacobs I, Ewesuedo R, Lula S, et al. Biosimilars for the Treatmen of Cancer: A Systematic Review of Published Evidence. BioDrugs. 2017;31(1):1-36. 